\title{
RESULTADOS FUNCIONALES DE LA ESTAPEDOTOMÍA EN PACIENTES CON OTOSCLEROSIS.
} HOSPITAL DE CLÍNICAS

\section{FUNCTIONAL RESULTS OF STAPEDOTOMY IN PATIENTS WITH OTOSCLEROSIS. HOSPITAL DE}

\author{
CLÍNICAS
}

\section{Raúl TORNACO ${ }^{1}$, Peter SCHWEISS ${ }^{1}$, Raquel TEJADA ${ }^{1}$, Jorge TORRES ${ }^{1}$}

${ }^{1}$ Universidad Nacional de Asunción, Facultad de Ciencias Médicas, San Lorenzo, Paraguay.

Cómo citar este artículo: Tornaco R, Schwiss P, Tejada R, Torres J. Resultados funcionales de la estapedotomía en pacientes con otosclerosis. Hospital de Clínicas. Med. clín. soc. 2019;3(3):88-94.

\section{RESUMEN}

Introducción: La otosclerosis es una enfermedad que produce remodelación anormal de hueso maduro y fijación de la platina del estribo a la ventana oval, produciendo hipoacusia conductiva. La estapedotomía es la técnica quirúrgica más utilizada. Tiene como objetivo aliviar la fijación de la cadena oscicular mediante una estapedotomía e inserción de una prótesis, con buenos resultados en manos expertas. Objetivo: Evaluar la variación audiométrica en pacientes operados de estapedotomía por otosclerosis. Metodología: Estudio observacional, descriptivo, de corte transversal retrospectivo, de serie de casos de pacientes con diagnóstico de Otosclerosis sometidos a estapedotomías en Hospital de Clínicas, desde el 2012 a 2016. Resultados: Se analizó un total de 14 fichas correspondientes a 11 pacientes operados en el periodo de enero 2012 a julio 2016 . El $72 \%$ fue de sexo femenino. La media de edades fue de $38,35 \pm 15$ años. Todos los pacientes presentaban hipoacusia. El $50 \%$ de las estapedotomías fue del lado derecho; 3 pacientes (27\%) fueron operados bilateralmente en dos tiempos. El 85,7\% presentaba afectación bilateral en la audiometría pre quirúrgica. La impedanciometría informó que el $71 \%$ presentó curva As bilateral; $14 \%$ curva As del oído derecho y 14\% curva A en ambos oídos y reflejo estapedial negativo bilateral en el 57 \%. En audiometría postquirúrgica se encontró una mejoría del 64 \% y una evolución de la enfermedad con empeoramiento de la hipoacusia en 21,4\%. Solo 2 pacientes presentaron complicaciones post quirúrgicas (1 fístula perilinfática y 1 desinserción de prótesis de yunque). Discusión: Acorde a la literatura la mayoría fue del sexo femenino, el rango etario coincide con estudios similares en la región, constatamos mejoría conductiva en la audiometría, pero un empeoramiento neurosensorial por la evolución de la enfermedad. La estapedotomía en nuestro estudio resultó una cirugía con buenos resultados y pocas complicaciones.

Palabras clave: Otosclerosis; Estapedotomía; Audiometría; Hipoacusia.

\section{ABSTRACT}

Introduction: Otosclerosis is a disease that produces abnormal remodeling of mature bone and fixation of the plate from the stapes to the oval window, producing conductive hearing loss. Stapedotomy is the most commonly used surgical technique. Its objective is to alleviate the fixation of the oscicular chain by means of a stapedotomy and insertion of a prosthesis, with good results in expert hands. Objective: To evaluate audiometric variation in patients operated for stapedotomy due to otosclerosis. Methodology: Observational, descriptive, retrospective cross-sectional, non-probabilistic sampling of consecutive cases of patients diagnosed with Otosclerosis who underwent stapedotomy at the Hospital de Clínicas, from 2012 to 2016. Results: We analyzed a total of 14 records corresponding to 11 patients operated in the period from January 2012 to July 2016. Seventy-two percent were female. The mean age was $38.35 \pm 15$ years. All patients had hearing loss. $50 \%$ of the stapedotomies were on the 
right side; 3 patients (27\%) were operated bilaterally in two stages. $85.7 \%$ had bilateral involvement in preoperative audiometry. Impedanceometry reported that $71 \%$ had bilateral as curve; $14 \%$ right ear As curve and $14 \% \mathrm{~A}$ curve in both ears and bilateral negative stapedial reflex in $57 \%$. In post-surgical audiometry an improvement of $64 \%$ was found, and a disease evolution with worsening of hearing loss in $21.4 \%$. Only 2 patients presented postsurgical complications ( 1 perilymphatic fistula and 1 anvil prosthesis disinsertion). Discussion: According to the literature the majority was female, the age range coincides with similar studies in the region, we found conductive improvement in audiometry, but a sensorineural worsening by the evolution of the disease. The stapedotomy in our study resulted in surgery with good results and few complications.

Keywords: Otosclerosis; Stapedotomy; Audiometry; Hearing loss.

\section{INTRODUCCIÓN}

La otosclerosis es una enfermedad localizada y primaria de la cápsula ótica y los huesecillos del oído. Produce una remodelación anormal de hueso maduro de la cápsula ótica por acción de osteoclastos y reemplazo por tejido óseo de gran espesor, celularidad y vascularización. Este fenómeno, denominado otoespongiosis, tiene predilección por el área anterior de la ventana oval (la fisura antefenestra está afectada en el $96 \%$ de los casos de otosclerosis del hueso temporal) (1), lo que lleva a la fijación de la platina del estribo a la ventana, consecuentemente una hipoacusia de tipo conductiva (2).

Su etiología sigue siendo poco conocida, sin embargo, está asociada a factores ambientales como las infecciones virales (virus del sarampión), el flúor, el estrógeno, además, de causas genéticas $(3,4)$. Esta enfermedad es dos veces más frecuente en mujeres que en hombres; la prevalencia aproximada es de $0,3 \%$ en la población blanca y mestiza. Es más frecuente la afectación bilateral que unilateral (la bilateral es en promedio $80 \%$ ) (5). Es de destacar que los antecedentes familiares se encuentran presentes en un tercio de los pacientes (6).

La presentación clínica característica de esta enfermedad es una hipoacusia de conducción, que se produce cuando el foco de otosclerosis compromete la platina del estribo y provoca la fijación de esta. También puede presentar un componente sensorioneural, histológicamente relacionado con una extensión medial del foco, hacia la cóclea y la deposición de colágeno en todo el ligamento espiral $(7,8)$.

La estapedectomía se introdujo hace más de 50 años, y desde esa fecha la técnica ha sufrido múltiples modificaciones. Actualmente la estapedotomía se ha convertido en la técnica quirúrgica más utilizada. La cirugía tiene como objetivo aliviar la fijación de la cadena oscicular mediante la realización de una estapedotomía e inserción de una prótesis para evitar la fijación del estribo (9). La cirugía muestra buenos resultados auditivos, disminuyendo la brecha aérea/ósea, y teniendo efecto favorable incluso en la disminución de otros síntomas como el acufeno (10), empero, es un procedimiento difícil y delicado, y no está exento de fallos en cuanto al procedimiento se refiere, por lo que requiere de manos expertas para su realización, con tasas de éxito que rondan el 96 \% en manos de cirujanos experimentados, con baja tasa de complicaciones y un resultado estable a largo plazo (11).

El paciente debe tener en cuenta que el procedimiento quirúrgico puede aliviar el síntoma que es la hipoacusia o el acufeno, pero el proceso vicioso sigue intacto, pudiendo llevar a un 
empeoramiento de la audición, ya sea conductiva, neurosensorial o mixta, incluso años después del procedimiento quirúrgico, o en el oído contralateral (12). El objetivo general de la investigación fue determinar los resultados funcionales de la estapedotomía en pacientes con otosclerosis.

\section{METOdOLOGÍA}

Estudio observacional, descriptivo de corte transversal y temporalmente retrospectivo, desarrollado en el Hospital de Clínicas en el año 2016 con muestro no probabilístico de casos consecutivos, la población accesible son los pacientes con diagnóstico de otosclerosis sometidos a estapedotomía en Hospital de Clínicas, Asunción-Paraguay, durante el periodo de 2012 a 2016, se incluyó a todos los pacientes con diagnóstico de otosclerosis sometidos a estapedotomía en el Hospital de Clínicas.

Los datos se recogieron a partir de las historias clínicas de la cátedra y servicio de Otorrinolaringología y Cirugía del Hospital de Clínicas, Asunción-Paraguay. Fuente de datos de segundo orden. Se incluyó 14 intervenciones. Las variables sociodemográficas fueron: sexo (hombre, mujer), edad (en años cumplidos), los datos clínicos del paciente: motivo de consulta, audiometría pre-quirúrgica, logoaudiometría pre-quirúrgica, impedanciometría pre-quirúrgica, audiometría posquirúrgica, logoaudiometría posquirúrgica, logoaudiometría posquirúrgica, tiempo transcurrido desde la cirugía hasta el estudio audiológico, complicaciones, evolución.

Se utilizó una fuente de datos secundaria, la cual correspondió a las fichas clínicas de la cátedra y servicio de Otorrinolaringología del Hospital de Clínicas, de pacientes sometidos a estapedotomía. Se incluyeron 14 intervenciones, en 11 pacientes, durante el periodo de enero de 2012 a julio de 2016. Se procedió a recolectar los datos de las fichas clínicas. Posteriormente fueron comparados según datos sociodemográficos y clínicos.

Se mantuvo la confidencialidad de identidad de los pacientes y se garantizó que la exposición de los resultados sea en forma colectiva o, en caso de que sea individual, manteniendo la privacidad del mismo. En caso de que se haya percatado información faltante, los investigadores se encargaran de informar a los pacientes y/o médicos encargados. Los datos obtenidos en esta encuesta no se utilizarán para otros fines ajenos a la investigación en este estudio. Todos los pacientes tuvieron la misma oportunidad de participar en el estudio. El desarrollo de este estudio se dio bajo autorización de la jefatura del servicio.

\section{RESULTADOS}

Se ha analizado un total de 14 fichas correspondientes a 11 pacientes operados en el periodo de enero 2012 a julio 2016. El 73 \% de los pacientes fue del sexo femenino. En cuanto a la edad, la media fue de $38 \pm 15$ años con un rango de 16 a 74 años.

El $100 \%$ de las cirugías practicadas fue estapedotomía, de las cuales: el $50 \%$ fue del lado derecho y el $50 \%$ fue del lado izquierdo; de estos, 3 pacientes ( $27 \%$ ) fueron operados en ambos oídos en tiempos operatorios distintos, con un intervalo promedio de 28 meses, entre ambos oídos.

En cuanto a los síntomas más frecuentes presentados por los pacientes se describe según la figura 1. 
FIGURA 1. MOTIVOS DE CONSULTA MÁS FRECUENTES EN LOS PACIENTES ESTUDIADOS (n=14)

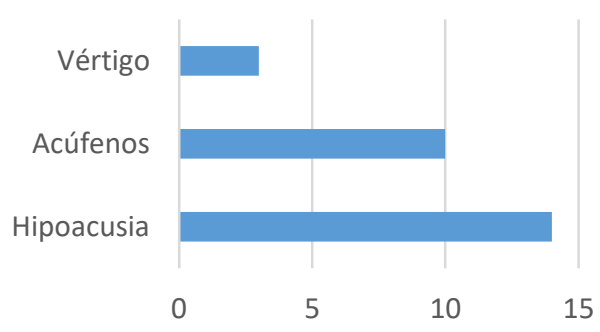

En la evaluación audiométrica prequirúrgica se encontró un 85,7 \% de pacientes con algún grado de afectación bilateral, la distribución encontrada se detalla en la tabla 1.

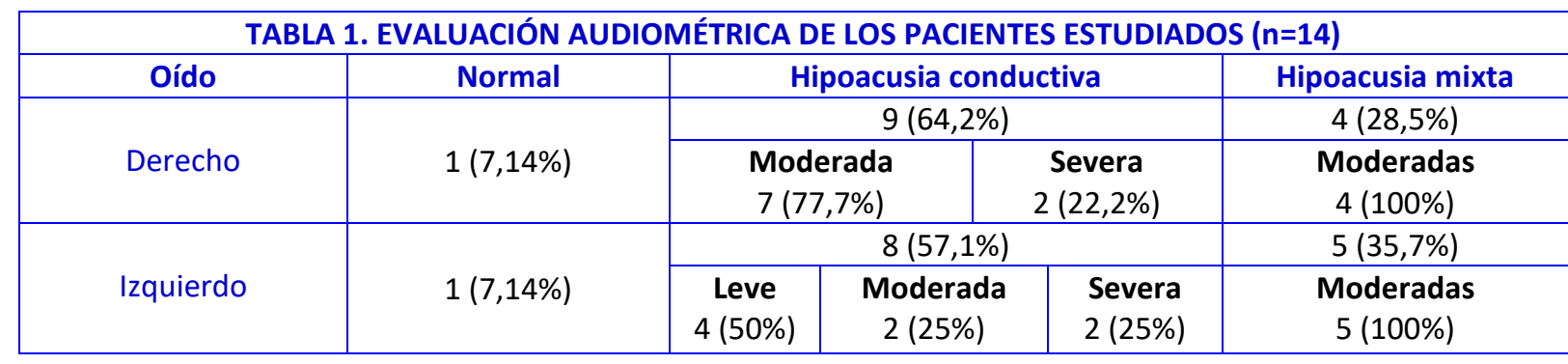

En cuanto a los datos hallados en la timpanometría, la impedanciometría informó que el $71 \%$ presentó curva As bilateral; $14 \%$ presentó curva As solamente del oído derecho y otro $14 \%$ curva $A$ en ambos oídos. Se informó además reflejo estapedial negativo bilateral en el $57 \%$. En la logoaudiometría pre - quirúrgica se constató lo mostrado en la tabla 2.

\begin{tabular}{|c|c|c|c|c|}
\hline \multicolumn{4}{|c|}{ TABLA 2. EVALUACIÓN DE LOGOAUDIOMETRÍA PRE-QUIRÚGICA DE LOS PACIENTES ESTUDIADOS (n=14) } \\
\hline Oído & Normal & Desplazada & Promedio & $\mathbf{9 0} \%$ de discriminación \\
\hline Derecho & $1(8,4 \%)$ & $11(91,6 \%)$ & $52 \mathrm{~dB}$ & $10(84 \%)$ \\
\hline Izquierdo & $3(25 \%)$ & $9(75 \%)$ & $43,8 \mathrm{~dB}$ & $10(84 \%)$ \\
\hline
\end{tabular}

Se realizó control audiométrico en un tiempo de 4,15 meses en promedio posterior a la cirugía, en los cuales se encontró una mejoría del $64 \%$, y una evolución de la enfermedad con empeoramiento de la hipoacusia en $21,4 \%$. Un solo paciente (7 \%) no realizó el estudio post cirugía. El 28,5 \% tuvo una caída en la transmisión neurosensorial. Los hallazgos se detallan en la tabla 3.

\begin{tabular}{|c|c|c|}
\hline \multirow{2}{*}{ Lado } & TABLA 3. CONTROL AUDIOMÉTRICO DE LOS PACIENTES ESTUDIADOS (n=14) & Post quirúrgico \\
\hline \multirow{3}{*}{ Derecha } & HA mixta moderada & HA mixta moderada \\
\cline { 2 - 3 } & HA conductiva moderada & Normal \\
\cline { 2 - 3 } & HA conductiva moderada & HA conductiva leve \\
\cline { 2 - 3 } & HA conductiva moderada & HA conductiva leve \\
\cline { 2 - 3 } & HA conductiva moderada & No realiza control \\
\cline { 2 - 3 } & HA conductiva moderada & HA mixta severa \\
\cline { 2 - 3 } & HA conductiva severa & HA conductiva moderada \\
\hline Izquierda & HA conductiva moderada & HA mixta severa \\
\cline { 2 - 3 } & HA conductiva severa & HA conductiva leve \\
\cline { 2 - 3 } & HA mixta moderada & Handa \\
\cline { 2 - 3 } & &
\end{tabular}




\begin{tabular}{|c|c|c|}
\hline \multirow{2}{*}{} & HA conductiva moderada & HA mixta leve \\
\cline { 2 - 3 } & HA mixta moderada & HA conductiva moderada \\
\cline { 2 - 3 } & HA mixta moderada & HA mixta leve \\
\hline HA mixta moderada & HA conductiva moderada \\
\hline
\end{tabular}

Se realizó logoaudiometría postquirúrgica y el hallazgo se ve en la tabla 4. 2 pacientes presentaron complicaciones post quirúrgicas (1 fístula perilinfática y 1 desinserción de prótesis de yunque).

\begin{tabular}{|c|c|c|c|c|}
\hline \multicolumn{5}{|c|}{ TABLA 4. LOGOAUDIOMETRÍA POSQUIRÚRGICA A LOS PACIENTES ESTUDIADOS (n=14) } \\
\hline Oído & Normal & Desplazada & Promedio & $>90 \%$ de discriminación \\
\hline Derecho & $2(33,3 \%)$ & $4(66,6 \%)$ & $52,4 \mathrm{~dB}$ & $4(66,6 \%)$ \\
\hline Izquierdo & 0 & $6(100 \%)$ & $45,8 \mathrm{~dB}$ & $4(66,6 \%)$ \\
\hline
\end{tabular}

\section{DISCUSIÓN}

Según la literatura consultada, esta patología presenta una prevalencia mayor en el sexo femenino; se encontró en esta investigación predominio de pacientes del sexo femenino con un $73 \%$, al consultar datos de estudios en la región, en el Hospital Clínico de la Universidad de Chile (13), se evidenció una afectación con predominancia del 81 \% de pacientes del sexo femenino, lo que concuerda con los hallazgos de la presente investigación.

El mencionado estudio presentó una media de edades de 47 años, rango de 33 a 66 años, números similares a los obtenidos en nuestro trabajo, con media de 38,35 años con un rango de 16 a 74 años, mientras que, en otro estudio de la misma Universidad, se reportó una media de 44,2 \pm 7,7 años (14), la literatura europea consultada refiere que la edad de presentación promedio es entre la segunda y cuarta década de la vida, quizás en nuestro medio el tiempo en el que acceden al tratamiento está más alejado del inicio de los síntomas (15).

La bilateralidad de la afección es reportada en más del $80 \%$ en el texto utilizado de base. En cuanto este punto en particular, $85,7 \%$ de pacientes en este estudio presentaba algún grado de afección bilateral, comparándolo con un estudio realizado en la Universidad de Chile, 62,31 \% tenía compromiso bilateral (16).

En nuestro estudio se logró una mejoría en los informes audiométricos del $64 \%$ de los pacientes sometidos a estapedotomía, no así en la logoaudiometría, con valores pre y postquirúrgicos casi iguales, sin embargo, un trabajo realizado en Francia comparando la eficacia de dos prótesis halló una mejoría media de, respectivamente, 16,63 dB en el grupo con un tipo SCP (titanio) de prótesis y 20,59 dB en el grupo prótesis PCP (fluoroplastic). Cabe destacar que en esta investigación la totalidad de las prótesis utilizadas eran de fluoroplastic (17).

Una pérdida significativa postoperatoria de audición neurosensorial fue resultado de un estudio (18), en esta investigación se constató en el $28,5 \%$ de los pacientes. En un estudio realizado en India se presentaron un total de $13 \%$ de complicaciones postquirúrgicas en comparación al 14,2 \% de la presente investigación (19).

\section{CONFLICTOS DE INTERÉS Y FUENTE DE FINANCIACIÓN}

Los autores declaran no poseer conflictos de interés. Fuente de financiación: ninguna. 


\section{REFERENCIAS BIBLIOGRÁFICAS}

1. Wichova H, Alvi S, Boatright C, Ledbetter L, Staecker H, Lin J. High-Resolution Computed Tomography of the Inner Ear: Effect of Otosclerosis on Cochlear Aqueduct Dimensions. Ann Otol Rhinol Laryngol. 2019;128(8):749-54. https://dx.doi.org/10.1177/0003489419842579

2. KJ L. Essential Otolaryngology, Head and Neck Surgery.9th edMc. Graw Hill; 2008.

3. Batson L, Rizzolo D. Otosclerosis: An update on diagnosis and treatment. JAAPA. 2017;30(2):17-22. https://dx.doi.org/10.1097/01.JAA.0000511784.21936.1b

4. Thys M, Van Camp G. Genetics of otosclerosis. Otol Neurotol. 2009;30(8):1021-32. https://dx.doi.org/10.1097/MAO.0b013e3181a86509

5. van Loon MC, Merkus P, Smit CF, Smits C, Witte BI, Hensen EF. Stapedotomy in cochlear implant candidates with far advanced otosclerosis: a systematic review of the literature and meta-analysis. Otol Neurotol. 2014;35(10):1707-14. https://dx.doi.org/10.1097/MA0.0000000000000637

6. Declau F, Van Spaendonck M, Timmermans JP, Michaels L, Liang J, Qiu JP, et al. Prevalence of Otosclerosis in an Unselected Series of Temporal Bones: Otology \& Neurotology. 2001;22(5):596-602. https://dx.doi.org/10.1097/00129492200109000-00006

7. Dwyer-Hemmings L, Manjaly JG, Nash R, Mukherjee A, Lavy JA. Stapes Surgery for Profound Hearing Loss Secondary to Otosclerosis: Ear, Nose Throat J. 2019;98(5):2738. https://dx.doi.org/10.1177/0145561319834540

8. Bouaity B, Chihani M, Touati M, Darouassi Y, Nadour K, Ammar H. L'otospongiose: étude rétrospective à propos de 36 cas. Pan Afr Med J. 2014;18:242. https://dx.doi.org/10.11604/pamj.2014.18.242.3509

9. Piñeyro Silva EE, Gutiérrez Espinosa CA. Estapedotomía convencional versus estapedotomía con láser $\mathrm{CO} 2$ en el Hospital Regional «General Ignacio Zaragoza». An Med Asoc Med Hosp ABC. 2018;62(4):256-60. URL.

10. Causse JR, Causse JB. Otospongiosis as a genetic disease. Early detection, medical management, and prevention. Am J Otol. enero de 1984;5(3):211-23. URL.

11. Quesnel AM, Ishai R, McKenna MJ. Otosclerosis: Temporal Bone Pathology. Otolaryngol Clin North Am. 2018;51(2):291-303. https://doi.org/10.1016/j.otc.2017.11.001

12. Costas L, Alarcón L, Toso S. Compendio Paraguayo de Otorrinolaringología. Asunción: Editorial Servilibro. 2015.

13. Stott C C, Royer F M, Olmedo O R, Martínez S C, Valdés P C, Ortúzar B L. Hearing results on stapedostomies in complicated platens. Rev Otorrinolaringol Cir Cabeza Cuello. 2006;66(2):89-94. https://dx.doi.org/10.4067/S0718-48162006000200003

14. Délano R PH, Alvo V A, Ojeda S A, Stott C C. Audiological outcome and surgical findings in patients with bilateral surgery for otosclerosis. Rev Otorrinolaringol Cir Cabeza Cuello. 2011;71(3):203-6. https://dx.doi.org/10.4067/s0718-48162011000300003

15. Stott C C, Ojeda S A, Muñoz S D, Moyano S L. Otosclerosis: Histopathological features and stapedostomy hearing results. Rev Otorrinolaringol Cir Cabeza Cuello. 2012;72(2):125-32. https://dx.doi.org/10.4067/S0718-48162012000200004

16. Potena $M$, Portmann D, Guindi S. Comparaison audiologique entre deux clips différents de prothèse dans la chirurgie d'étrier. Rev Laryngol Otol Rhinol Bord. 2015;136(1):33-6. URL. 
17. Vincent R, Sperling NM, Oates J, Jindal M. Surgical findings and long-term hearing results in 3,050 stapedotomies for primary otosclerosis: a prospective study with the otology-neurotology database. Otol Neurotol. 2006;27(8 Suppl 2):S25-47. https://dx.doi.org/10.1097/01.mao.0000235311.80066.df

18. 1.Adedeji TO, Indorewala S, Indorewala A, Nemade G. Stapedotomy and its effect on hearing - our experience with 54 cases. Afr H Sci. 2016;16(1):276. https://dx.doi.org/10.4314/ahs.v16i1.36 